\title{
Comparing the Effects of Leadership and Organizational Culture on Innovation in Technology-Based Organizations and Other Industries
}

\author{
Eva Petiz LOUSÃ \\ CEOS.PP / ISCAP / P.PORTO, Rua Jaime Lopes Amorim s/n, 4465-004 \\ Matosinhos, Portugal \\ evapetiz@iscap.ipp.pt
}

Received date:14 November 2019; Accepted date: 31 May 2020; Published date: 27 July 2020

Copyright (C) 2020. Eva Petiz LOUSÃ. Distributed under Creative Commons Attribution 4.0 International CC-BY 4.0

\begin{abstract}
The relationship between leadership, organizational culture and innovation is examined. One hundred and two Portuguese organizations from different activities sectors participated in the study. In each organization, top managers were asked about the innovation activity and employees were asked about the leadership and organizational culture of their organizations. Organizations were classified as either technology-based or non-technology-based. A structural model was tested by multi-group analysis, considering the sector of activity, technology-based companies and non-technology-based companies. Results show significant differences in the relationship between leadership and organizational culture in the prediction of innovation and on the contribution of the several variables in the model, suggesting that the activity sector of an organization acts as a moderator of that relationship. Some contributions are highlighted.
\end{abstract}

Keywords: Organizational culture, transformational leadership, organizational innovation, technology-based organizations and activity sector.

\section{Introduction and theoretical framework}

Leadership, organizational culture and innovation are mentioned in the literature as key factors for the success of organizations and their competitive advantage. Some studies have shown that transformational leadership supports the organizational innovation (e.g. Gumusluoglu and Ilsev, 2009; Jung, Chow and Wu, 2008; Jung, Wu and Chow, 2003; Mumford, Scott, Gaddis and Strange, 2002).
Others' research has asserted that organizational culture is an important aspect of organizational life that stimulates creativity and innovation (Amabile et al., 1996; Chandler, Keller and Lyon; 2000; Martins and Terblanche, 2003). Moreover, Bass and Avolio (1993) have argued that transformational leadership and organizational culture are interconnected factors, such that transformational leaders working in the existing culture are concerned with changing organizational

Cite this Article as: Eva Petiz LOUSÃ (2020)," Comparing the Effects of Leadership and Organizational Culture on Innovation in Technology-Based Organizations and Other Industries", IBIMA Business Review, Vol. 2020 (2020), Article ID 315185, DOI: 10.5171/2020.315185 
culture and the organization culture also affects leadership.

The present study examines the relationships between leadership, organizational culture and innovation, and it aims to examine this relationship according to the company's technological sector. Recently, the authors have noticed an exponential increase of companies in Portugal that base their activity on the intensive use of the technological knowledge. These types of organizations technology-based organizations - guide their activity according to market driven technological innovation principles, dedicated to the development of production and commercialization of innovative products/services (OECD, 2015). Although the use of knowledge and technology may be more intensive in some activity sectors than in others, it is recognized that the innovation may occur in any activity sector (OECD, 2005, 2015), and also in more traditional sectors. In this context, it is also relevant to analyze the innovation activity in companies from different sectors to compare and understand their activity in each activity sector.

Transformational leadership theory emphasizes the role of leaders in promoting change in their employees and organization (Bass and Riggio, 2006). Transformational leaders are typically described as those who align the individual, leader, group and organization's interests and objectives by providing an inspiring vision of the future (Bass, 1999). This type of leadership has been conceptualized in four dimensions: inspirational motivation (i.e. the leader communicates clearly and inspires workers to achieve important organizational goals); idealized influence (i.e. the leader provides a role model for followers and displays in them strong ethical principles); individualized consideration (i.e. the leader treats each follower as an individual with unique needs and attends to these needs); and intellectual stimulation (i.e. the leader encourages the follower to be creative and challenges him or her to think of old problems in new ways) (Bass and Riggio,
2006). In doing so, their role is to stimulate, inspire and support the growth and development of their followers as leaders (Bass, 1999; Bass and Riggio, 2006) and to encourage the innovation in the organization (Gumusluoglu and Ilsev, 2009; Jung et al, 2008; Mumford et al., 2002).

Organizational culture is understood as the basic assumptions that a group invented, discovered, or developed by learning to deal with their problems of external adaptation and internal integration (Schein, 1983, 1992). Based on this central assumption, Denison and collaborators (Denison, Nieminen, and Kotrba, 2014) develop an organizational culture model that enables the diagnosis and comparison of cultures. The model proposes the existence of four dimensions: Involvement (i.e. how much the members of the organization are involved in the management process), Consistency (i.e. the sharing of core values, consensus and common goals and objectives among members at all levels of the organization), Adaptability (i.e. the ability of organizations to adapt quickly to changing their environment, taking risks, learning from their mistakes, and adding value to their customers, seeing their opportunities to survive and grow) and Mission (i.e. the organization's strategic direction, purpose which allows it to set its global goals, as well as expressing the vision to its members). These traits were related to performance and organizational effectiveness (Denison et al, 2014), and the present study will relate them to innovation.

Concerning innovation, the literature has shown that it is a broad domain (Damanpour and Schneider, 2006; Lousã and Gomes, 2017; Ramos, Anderson, Peiró, and Zijlstra, 2016), so that the authors of this paper will only highlight how they understand innovation in the present study. Amabile et al. (1996) have defined the innovation based on two processes: the generation and subsequent implementation of new or significantly improved ideas. According to this conception, innovation has implied a 
creative process, i.e. the generation of new and useful ideas. The OECD (2005) highlights the implementation process extended to a new or significantly improved product (good or service) or process, a new marketing method or a new organizational method in business practices, workplace organization or external relations. The present study adopts both conceptualizations and analyzes the innovation activity of the company, based on the three dimensions of Lousã's model (2013): resources, processes and innovation results. The Resources refer to the inputs for the organization towards the activities for innovation (e.g. resources available, the level of qualifications and the participation in lifelong learning activities of the human resources, investment/expenditures in Research and Development). The Processes refer to those processes relevant to the innovative dynamic (e.g. partnerships, cooperation networks and protection and enhancement of knowledge). And the Results refer to the outcomes of the activities of a company (e.g. the turnover in the last three years; the evolution of sales of new products/services or significant improvements over the last three years; the ideas generated in the company transformed into innovative designs and the enterprise image). These indicators allow comparing the innovation activities between companies and appreciating the innovation dynamics within each company.

Based on this theoretic framework and in previous research of Lousã and Monico (2018) that explores the relation between these three constructs, taking the companies' size into account, this relationship is analysed in the present study, but comparing between the companies' industries. The hypothesis is tested that the technological sector will produce a moderating role in the relationship between leadership, culture and innovation. Therefore, the exploratory model starts from the interdependence between culture and leadership and analyses the influence of this association in innovation, considering the activity sector of the company (technology-based or nontechnology-based).

\section{Method \\ Sample}

A total of 102 Portuguese organizations participated in the study, 51 out of which belong to the technological sector (e.g., pharmacy; computer/information systems; biotechnology; electronics) and 51 to other activity sectors (e.g., textile; food products; paper products; wood). Regarding the dimension of the sample, there are $30.4 \%$ micro-enterprises, $38.2 \%$ small companies; $24.5 \%$ medium-sized companies and 5.9\% large companies, according to the European classification. These percentages are close to the enterprises' distribution in Portugal, since it is mainly made up of micro, small and medium enterprises. The age of the companies varies between two and 115 years $(\mathrm{M}=18.98$ years, $\mathrm{SD}=18.97$ years) and the number of employees per company ranges from two to $643(\mathrm{M}=$ 61.51 per company, $\mathrm{SD}=114.86)$. Two different questionnaires were administrated. Top managers of each organization $(\mathrm{N}=102)$ answered a questionnaire concerning the innovation activity in their organizations, whereas workers of each organization $(\mathrm{N}=854)$ answered a questionnaire concerning the leadership and culture of their organizations.

\section{Measures}

Transformational Leadership was measured with 17 items inspired by the four components of transformational leadership scale (Bass, 1985). Response options ranged from 1 (strongly disagree) to 5 (strongly agree). Confirmatory Factor Analysis (CFA) showed a unidimensional measure with an acceptable fit, $\mathrm{CFI}=.91$, $\chi 2 / \mathrm{df}=5.61$ and RMSEA=.08 (CI. 90 between .075 and .89 . Reliability was very $\operatorname{good}(\alpha=.96)$.

Organizational Culture was measured with 60 items from Organizational Culture Questionnaire Denison (Denison et al, 2014). Response options ranged from 1 (strongly disagree) to 5 (strongly agree). Reliability was very good ( $\alpha$ between .63 and .87). The structure of four dimensions 
and twelve indicators showed a good fit, $\chi 2 / \mathrm{df}=3.22$, CFI $=.85$ and $\mathrm{RMSEA}=.054$ (CI. 90 between .052 and .055 ).

Innovation was operationalized through the calculation of an index of innovation, constructed from several measures that are best likely to represent the construct of innovation (Bollen, 1989; Hoyle, 1995, Loehlin, 1997). The individual indicators were standardized and then aggregated by their sum, obtaining an unweighted " $\mathrm{z}$ Score". The individual measures and their operationalization of the Innovation index were: Company image (image), a 6-items scale concerning the contributions that innovation activities bring to the organization in terms of production of goods or services, clients in the face of competition and job creation, among others aspects (e.g. "innovation activities contribute to attracting more customers in the face of competition). Response options ranged from (much worse) to five (much better). Reliability was good ( $\alpha=.82)$. Resources made available (resources) was the 5-item subscale of climate innovation (Scott and Bruce, 1994). Response options ranged from (1) ' totally disagree' to (5) ' totally agree'. An example of an item is "in this organization, there are adequate resources dedicated to innovation". Reliability was $\alpha=.74$. Level of qualifications was measured by the years of education. Lifelong learning/ Training activities were measured by the percentage of workers who participated in lifelong learning activities and who are innovation oriented, concerning the previous year. These data were obtained in official documents. Investment in Research and Development was measured by the investment or expenditures made by the company in $R \& D$ in the last three years. These data were obtained from official documents. Partnerships and Cooperation was measured by the type of the company's cooperation networks with other entities towards innovation (e.g. competitors, suppliers, customers, universities, higher education institutions, $R \& D$ units). Networks were measured by the type of company's networks (e.g. international cooperation networks). Protection and enhancement of knowledge was measured by the ways that the company usually used to protect and enhance knowledge, (e.g. patenting). Ideas transformed into innovative projects were measured by the percentage of ideas generated in this company and transformed into innovative projects. Turnovers were measured by the evolution of the growth or decline of an activity in the last three years. These data were obtained from official documents. And finally, Volume of sales of new products/services or those that significantly improved, considering the past three years. The type of response adopted considers a scale with three options: Decreased (1), Same (2), Increased (3).

Technology - The manager was asked if the company was technology-based or nontechnology-based and in what sector.

\section{Procedures and Data Analysis}

Data were collected considering ethical issues such as participants' anonymity and confidentiality, and to avoid bias. Top management of each organization evaluated the innovation activity through a questionnaire once they were expected to be knowledgeable regarding the resources, processes and results of the innovation of their organizations. Employees from different parts of the organization, considering their representativeness in the organizational structure and the departments involved in the organization, evaluated the leadership and organizational culture in each company.

Data were analyzed with the statistical program SPSS and AMOS 24.0 (for Windows operating system). Confirmatory Factor Analysis was performed with AMOS. The structural equation model was established and specified according to literature (Byrne, 2010; Schumacker and Lomax, 2010).

\section{Results}

Descriptive statistics and intercorrelations of measures of Leadership, Innovation, and Culture and their dimensions are reported in Table 1. The association between Leadership and Innovation was moderate 
$(\mathrm{r}=.33)$ and with leadership and the total score Culture $(\mathrm{r}=.45)$; the correlation between Innovation and Culture was small (r=.18) (Cohen, 1988). 
Table 1: Descriptive statistics and intercorrelation matrix of measures of Leadership, Innovation, and Culture and their dimensions

\begin{tabular}{|c|c|c|c|c|c|c|c|c|c|c|c|c|c|c|c|c|}
\hline & M & SD & 1 & 2 & 3 & 4 & 5 & 6 & 7 & 8 & 9 & 10 & 11 & 12 & 13 & 14 \\
\hline 1. Leadership & 3.27 & 0.36 & 1 & & & & & & & & & & & & & \\
\hline 2. Culture & 15.43 & 2.37 & $\begin{array}{l}.45 \\
* *\end{array}$ & 1 & & & & & & & & & & & & \\
\hline $\begin{array}{l}\text { 3. Involvemen } \\
t\end{array}$ & 4.03 & 0.64 & $\begin{array}{l}.44 \\
* * \\
\end{array}$ & $\begin{array}{l}.97 \\
* *\end{array}$ & 1 & & & & & & & & & & & \\
\hline $\begin{array}{l}\text { 4. Team } \\
\text { Orientation }\end{array}$ & 3.49 & 0.57 & $\begin{array}{l}.42 \\
* *\end{array}$ & $\begin{array}{l}.95 \\
* *\end{array}$ & $\begin{array}{l}.99 \\
* *\end{array}$ & 1 & & & & & & & & & & \\
\hline $\begin{array}{l}\text { 5. Capability } \\
\text { Development }\end{array}$ & 4.02 & 0.70 & $\begin{array}{l}.44 \\
* * \\
\end{array}$ & $\begin{array}{l}.93 \\
* * \\
\end{array}$ & $\begin{array}{l}.97 \\
* * \\
\end{array}$ & $\begin{array}{l}.95 \\
* * \\
\end{array}$ & 1 & & & & & & & & & \\
\hline 6. Consistency & 3.38 & 0.52 & $\begin{array}{l}.44 \\
* *\end{array}$ & $\begin{array}{l}.99 \\
* *\end{array}$ & $\begin{array}{l}.98 \\
* *\end{array}$ & $\begin{array}{l}.96 \\
* *\end{array}$ & $\begin{array}{l}.94 \\
* *\end{array}$ & 1 & & & & & & & & \\
\hline 7. Values & 3.56 & 0.54 & $\begin{array}{l}.44 \\
* *\end{array}$ & $\begin{array}{l}.96 \\
* *\end{array}$ & $\begin{array}{l}.95 \\
* *\end{array}$ & $\begin{array}{l}.92 \\
* *\end{array}$ & $\begin{array}{l}.91 \\
* *\end{array}$ & $\begin{array}{l}.98 \\
* *\end{array}$ & 1 & & & & & & & \\
\hline 8. Agreement & 3.95 & 0.62 & $\begin{array}{l}.43 \\
* *\end{array}$ & $\begin{array}{l}.97 \\
* *\end{array}$ & $\begin{array}{l}.96 \\
* *\end{array}$ & $\begin{array}{l}.94 \\
* *\end{array}$ & $\begin{array}{l}.92 \\
* *\end{array}$ & $\begin{array}{l}.99 \\
* *\end{array}$ & $\begin{array}{l}.98 \\
* *\end{array}$ & 1 & & & & & & \\
\hline $\begin{array}{l}\text { 9. Coordinatio } \\
n \text { and } \\
\text { Integration }\end{array}$ & 3.33 & 0.55 & $\begin{array}{l}.40 \\
* *\end{array}$ & $\begin{array}{l}.94 \\
* *\end{array}$ & $\begin{array}{l}.92 \\
* *\end{array}$ & $\begin{array}{l}.90 \\
* *\end{array}$ & $\begin{array}{l}.87 \\
* *\end{array}$ & $\begin{array}{l}.96 \\
* *\end{array}$ & $\begin{array}{l}.92 \\
* *\end{array}$ & $\begin{array}{c}.94 \\
* *\end{array}$ & 1 & & & & & \\
\hline $\begin{array}{l}\text { 10. Adaptabili } \\
\text { ty }\end{array}$ & 3.89 & 0.58 & $\begin{array}{l}.44 \\
* * \\
\end{array}$ & $\begin{array}{l}.99 \\
* * \\
\end{array}$ & $\begin{array}{l}.95 \\
* * \\
\end{array}$ & $\begin{array}{l}.92 \\
* * \\
\end{array}$ & $\begin{array}{l}.91 \\
* * \\
\end{array}$ & $\begin{array}{l}.98 \\
* * \\
\end{array}$ & $\begin{array}{l}.95 \\
* * \\
\end{array}$ & $\begin{array}{l}.96 \\
* * \\
\end{array}$ & $\begin{array}{l}.92 \\
* * \\
\end{array}$ & 1 & & & & \\
\hline $\begin{array}{l}\text { 11. Creating } \\
\text { Change }\end{array}$ & 3.97 & 0.61 & $\begin{array}{l}.43 \\
* *\end{array}$ & $\begin{array}{l}.96 \\
* *\end{array}$ & $\begin{array}{l}.92 \\
* *\end{array}$ & $\begin{array}{l}.89 \\
* *\end{array}$ & $\begin{array}{l}.88 \\
* *\end{array}$ & $\begin{array}{l}.95 \\
* *\end{array}$ & $\begin{array}{l}.92 \\
* *\end{array}$ & $\begin{array}{l}.92 \\
* *\end{array}$ & $\begin{array}{l}.89 \\
* *\end{array}$ & $\begin{array}{l}.98 \\
* *\end{array}$ & 1 & & & \\
\hline $\begin{array}{l}\text { 12. Customer } \\
\text { Focus }\end{array}$ & 2.56 & 0.36 & $\begin{array}{l}.36 \\
* * \\
\end{array}$ & $\begin{array}{l}.91 \\
* * \\
\end{array}$ & $\begin{array}{l}.86 \\
* * \\
\end{array}$ & $\begin{array}{l}.83 \\
* * \\
\end{array}$ & $\begin{array}{l}.81 \\
* * \\
\end{array}$ & $\begin{array}{l}.89 \\
* * \\
\end{array}$ & $\begin{array}{l}.86 \\
* * \\
\end{array}$ & $\begin{array}{l}.87 \\
* * \\
\end{array}$ & $\begin{array}{l}.85 \\
* * \\
\end{array}$ & $\begin{array}{l}.94 \\
* * \\
\end{array}$ & $\begin{array}{l}.91 \\
* * \\
\end{array}$ & 1 & & \\
\hline $\begin{array}{l}\text { 13. Organizati } \\
\text { onal Learning }\end{array}$ & 3.73 & 0.55 & $\begin{array}{l}.43 \\
* * \\
\end{array}$ & $\begin{array}{l}.97 \\
* * \\
\end{array}$ & $\begin{array}{l}.92 \\
* * \\
\end{array}$ & $\begin{array}{l}.89 \\
* * \\
\end{array}$ & $\begin{array}{l}.88 \\
* * \\
\end{array}$ & $\begin{array}{l}.95 \\
* * \\
\end{array}$ & $\begin{array}{l}.92 \\
* * \\
\end{array}$ & $\begin{array}{l}.92 \\
* * \\
\end{array}$ & $\begin{array}{l}.89 \\
* * \\
\end{array}$ & $\begin{array}{l}.99 \\
* * \\
\end{array}$ & $\begin{array}{l}.96 \\
* * \\
\end{array}$ & $\begin{array}{l}.91 \\
* * \\
\end{array}$ & 1 & \\
\hline 14. Mission & 4.13 & 0.68 & $\begin{array}{l}.43 \\
* *\end{array}$ & $\begin{array}{l}.97 \\
* *\end{array}$ & $\begin{array}{l}.89 \\
* *\end{array}$ & $\begin{array}{l}.85 \\
* *\end{array}$ & $\begin{array}{l}.85 \\
* *\end{array}$ & $\begin{array}{l}.94 \\
* *\end{array}$ & $\begin{array}{l}.91 \\
* *\end{array}$ & $\begin{array}{l}.91 \\
* *\end{array}$ & $\begin{array}{l}.88 \\
* *\end{array}$ & $\begin{array}{l}.97 \\
* *\end{array}$ & $\begin{array}{l}.93 \\
* *\end{array}$ & $\begin{array}{l}.90 \\
* *\end{array}$ & $\begin{array}{l}.94 \\
* *\end{array}$ & 1 \\
\hline $\begin{array}{l}\text { 15. Strategic } \\
\text { Direction and } \\
\text { Intent }\end{array}$ & 4.07 & 0.75 & $\begin{array}{l}.42 \\
* *\end{array}$ & $\begin{array}{l}.89 \\
* *\end{array}$ & $\begin{array}{l}.79 \\
* *\end{array}$ & $\begin{array}{l}.76 \\
* *\end{array}$ & $\begin{array}{l}.76 \\
* *\end{array}$ & $\begin{array}{l}.85 \\
* *\end{array}$ & $\begin{array}{l}.83 \\
* *\end{array}$ & $\begin{array}{l}.82 \\
* *\end{array}$ & $\begin{array}{l}.79 \\
* *\end{array}$ & $\begin{array}{l}.89 \\
* *\end{array}$ & $\begin{array}{l}.85 \\
* *\end{array}$ & $\begin{array}{l}.81 \\
* *\end{array}$ & $\begin{array}{l}.86 \\
* *\end{array}$ & $\begin{array}{l}.94 \\
* *\end{array}$ \\
\hline $\begin{array}{l}\text { 16. Goals and } \\
\text { Objectives }\end{array}$ & 3.67 & 0.63 & $\begin{array}{l}.40 \\
* * \\
\end{array}$ & $\begin{array}{l}.94 \\
* *\end{array}$ & $\begin{array}{l}.86 \\
* *\end{array}$ & $\begin{array}{l}.82 \\
* * \\
\end{array}$ & $\begin{array}{l}.82 \\
* * \\
\end{array}$ & $\begin{array}{l}.91 \\
* *\end{array}$ & $\begin{array}{l}.88 \\
* * \\
\end{array}$ & $\begin{array}{l}.88 \\
* *\end{array}$ & $\begin{array}{l}.86 \\
* * \\
\end{array}$ & $\begin{array}{l}.94 \\
* *\end{array}$ & $\begin{array}{l}.90 \\
* *\end{array}$ & $\begin{array}{l}.88 \\
* *\end{array}$ & $\begin{array}{l}.91 \\
* *\end{array}$ & $\begin{array}{l}.99 \\
* *\end{array}$ \\
\hline 17. Vision & 3.68 & 0.62 & $\begin{array}{l}.42 \\
* * \\
\end{array}$ & $\begin{array}{l}.95 \\
* * \\
\end{array}$ & $\begin{array}{l}.86 \\
* * \\
\end{array}$ & $\begin{array}{l}.83 \\
* * \\
\end{array}$ & $\begin{array}{l}.82 \\
* * \\
\end{array}$ & $\begin{array}{l}.91 \\
* *\end{array}$ & $\begin{array}{l}.88 \\
* * \\
\end{array}$ & $\begin{array}{l}.89 \\
* * \\
\end{array}$ & $\begin{array}{l}.86 \\
* * \\
\end{array}$ & $\begin{array}{l}.95 \\
* * \\
\end{array}$ & $\begin{array}{l}.91 \\
* *\end{array}$ & $\begin{array}{l}.88 \\
* * \\
\end{array}$ & $\begin{array}{l}.92 \\
* * \\
\end{array}$ & $\begin{array}{l}.99 \\
* * \\
\end{array}$ \\
\hline $\begin{array}{l}\text { 18. Innovatio } \\
\text { n (sum) }\end{array}$ & 1.51 & 4.55 & $\begin{array}{l}.33 \\
* * \\
\end{array}$ & $\begin{array}{l}.18 \\
* *\end{array}$ & $\begin{array}{l}.17 \\
* *\end{array}$ & $\begin{array}{l}.15 \\
* * \\
\end{array}$ & $\begin{array}{l}.17 \\
* * \\
\end{array}$ & $\begin{array}{l}.18 \\
* * \\
\end{array}$ & $\begin{array}{l}.20 \\
* * \\
\end{array}$ & $\begin{array}{l}.18 \\
* * \\
\end{array}$ & $\begin{array}{l}.17 \\
* * \\
\end{array}$ & $\begin{array}{l}.19 \\
* * \\
\end{array}$ & $\begin{array}{l}.19 \\
* *\end{array}$ & $\begin{array}{l}.21 \\
* *\end{array}$ & $\begin{array}{l}.19 \\
* * \\
\end{array}$ & $\begin{array}{l}.17 \\
* * \\
\end{array}$ \\
\hline $\begin{array}{l}\text { 19. Resources } \\
\text { made } \\
\text { available }\end{array}$ & 3.70 & 0.67 & $\begin{array}{l}.37 \\
* *\end{array}$ & $\begin{array}{l}.20 \\
* *\end{array}$ & $\begin{array}{l}.18 \\
* *\end{array}$ & $\begin{array}{l}.16 \\
* *\end{array}$ & $\begin{array}{l}.18 \\
* *\end{array}$ & $\begin{array}{l}.19 \\
* *\end{array}$ & $\begin{array}{l}.20 \\
* *\end{array}$ & $\begin{array}{l}.19 \\
* *\end{array}$ & $\begin{array}{l}.15 \\
* *\end{array}$ & $\begin{array}{l}.21 \\
* *\end{array}$ & $\begin{array}{l}.23 \\
* *\end{array}$ & $\begin{array}{l}.17 \\
* *\end{array}$ & $\begin{array}{l}.22 \\
* *\end{array}$ & $\begin{array}{l}.20 \\
* *\end{array}$ \\
\hline $\begin{array}{l}\text { 20. Level of } \\
\text { qualifications }\end{array}$ & 3.71 & 1.52 & $\begin{array}{l}18 \\
* * \\
\end{array}$ & $\begin{array}{l}.15 \\
* * \\
\end{array}$ & $\begin{array}{l}.19 \\
* * \\
\end{array}$ & $\begin{array}{l}.19 \\
* * \\
\end{array}$ & $\begin{array}{l}.21 \\
* * \\
\end{array}$ & $\begin{array}{l}.15 \\
* * \\
\end{array}$ & $\begin{array}{l}.15 \\
* * \\
\end{array}$ & $\begin{array}{l}.15 \\
* * \\
\end{array}$ & $\begin{array}{l}.12 \\
* * \\
\end{array}$ & $\begin{array}{l}.13 \\
* * \\
\end{array}$ & $\begin{array}{l}.13 \\
* * \\
\end{array}$ & $\begin{array}{l}.10 \\
* * \\
\end{array}$ & $\begin{array}{l}.13 \\
* * \\
\end{array}$ & $\begin{array}{l}.10 \\
* * \\
\end{array}$ \\
\hline $\begin{array}{l}\text { 21. Lifelong } \\
\text { learning/Trai } \\
\text { ning activities }\end{array}$ & 3.22 & 1.30 & $\begin{array}{l}.09 \\
*\end{array}$ & .06 & .04 & .04 & .05 & .05 & .05 & .04 & .03 & $\begin{array}{c}.07 \\
*\end{array}$ & .06 & $\begin{array}{c}.09 \\
*\end{array}$ & $\begin{array}{l}.07 \\
*\end{array}$ & $\begin{array}{c}.08 \\
*\end{array}$ \\
\hline $\begin{array}{l}\text { 22. Partnersh } \\
\text { ips and } \\
\text { Cooperation }\end{array}$ & 3.88 & 1.99 & $\begin{array}{l}.11 \\
* *\end{array}$ & $\begin{array}{c}.09 \\
*\end{array}$ & $\begin{array}{l}.10 \\
* *\end{array}$ & $\begin{array}{c}.08 \\
*\end{array}$ & $\begin{array}{l}.11 \\
* *\end{array}$ & $\begin{array}{c}.09 \\
*\end{array}$ & $\begin{array}{l}.09 \\
* *\end{array}$ & $\begin{array}{l}.09 \\
* *\end{array}$ & .06 & $\begin{array}{c}.09 \\
*\end{array}$ & $\begin{array}{l}.09 \\
* *\end{array}$ & .06 & $\begin{array}{c}.09 \\
*\end{array}$ & $\begin{array}{c}.07 \\
*\end{array}$ \\
\hline $\begin{array}{l}\text { 23. Networki } \\
\text { ng }\end{array}$ & 0.96 & 1.09 & .05 & .04 & .05 & .05 & $\begin{array}{c}.08 \\
* \\
\end{array}$ & .04 & .05 & .04 & .01 & .03 & .01 & .01 & .04 & .02 \\
\hline $\begin{array}{l}\text { 24. Knowledg } \\
\text { e protection }\end{array}$ & 2.01 & 1.63 & $\begin{array}{l}- \\
.14 \\
* *\end{array}$ & $\begin{array}{c}- \\
.05\end{array}$ &. &. &. &. & $\begin{array}{c}- \\
.07\end{array}$ & $\begin{array}{c}- \\
.06\end{array}$ & $\begin{array}{c}- \\
.08 \\
*\end{array}$ & $\begin{array}{c}- \\
.05\end{array}$ &. & $\begin{array}{c}- \\
.04\end{array}$ & $\begin{array}{c}- \\
.05\end{array}$ & $\begin{array}{c}- \\
.04\end{array}$ \\
\hline $\begin{array}{l}\text { 25. Ideas } \\
\text { transformed } \\
\text { into } \\
\text { innovative } \\
\text { projects }\end{array}$ & 2.68 & 0.86 & $\begin{array}{l}.27 \\
* *\end{array}$ & $\begin{array}{l}.19 \\
* *\end{array}$ & $\begin{array}{l}.15 \\
* *\end{array}$ & $\begin{array}{l}.14 \\
* *\end{array}$ & $\begin{array}{l}.15 \\
* *\end{array}$ & $\begin{array}{l}.17 \\
* *\end{array}$ & $\begin{array}{l}.18 \\
* *\end{array}$ & $\begin{array}{l}.17 \\
* *\end{array}$ & $\begin{array}{l}.13 \\
* *\end{array}$ & $\begin{array}{l}.20 \\
* *\end{array}$ & $\begin{array}{l}.21 \\
* *\end{array}$ & $\begin{array}{l}.15 \\
* *\end{array}$ & $\begin{array}{l}.20 \\
* *\end{array}$ & $\begin{array}{l}.22 \\
* *\end{array}$ \\
\hline
\end{tabular}




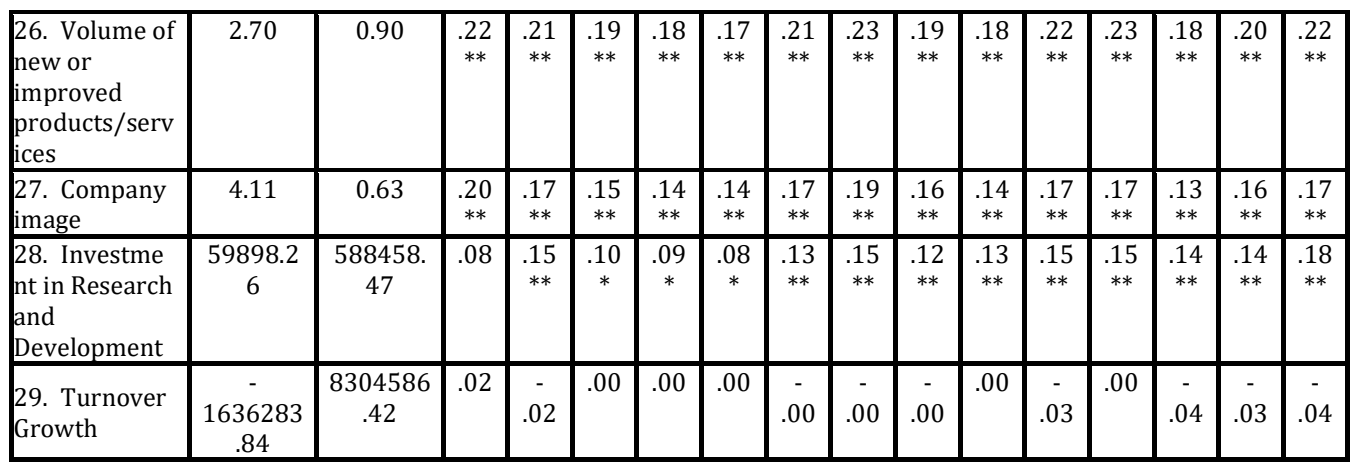

Note:*. Correlation is significant at the 0.05 level (2-tailed).; **. Correlation is significant at the 0.01 level (2tailed)

Table 1: Descriptive statistics and intercorrelation matrix of measures of Leadership, Innovation, and Culture and their dimensions (cont.)

\begin{tabular}{|c|c|c|c|c|c|c|c|c|c|c|c|c|c|c|c|c|c|}
\hline & $\mathbf{M}$ & SD & 15 & 16 & 17 & 18 & 19 & 20 & 21 & 22 & 23 & 24 & 25 & 26 & $\begin{array}{l}2 \\
7\end{array}$ & $\begin{array}{l}2 \\
8\end{array}$ & $\begin{array}{l}2 \\
9\end{array}$ \\
\hline 1. Leadership & 3.27 & 0.36 & & & & & & & & & & & & & & & \\
\hline 2. Culture & 15.43 & 2.37 & & & & & & & & & & & & & & & \\
\hline $\begin{array}{l}\text { 3. Involvemen } \\
t\end{array}$ & 4.03 & 0.64 & & & & & & & & & & & & & & & \\
\hline $\begin{array}{l}\text { 4. Team } \\
\text { Orientation }\end{array}$ & 3.49 & 0.57 & & & & & & & & & & & & & & & \\
\hline $\begin{array}{l}\text { 5. Capability } \\
\text { Development }\end{array}$ & 4.02 & 0.70 & & & & & & & & & & & & & & & \\
\hline 6. Consistency & 3.38 & 0.52 & & & & & & & & & & & & & & & \\
\hline 7.Values & 3.56 & 0.54 & & & & & & & & & & & & & & & \\
\hline 8. Agreement & 3.95 & 0.62 & & & & & & & & & & & & & & & \\
\hline $\begin{array}{l}\text { 9. Coordinatio } \\
n \text { and } \\
\text { Integration }\end{array}$ & 3.33 & 0.55 & & & & & & & & & & & & & & & \\
\hline $\begin{array}{l}\text { 10. Adaptabil } \\
\text { ity }\end{array}$ & 3.89 & 0.58 & & & & & & & & & & & & & & & \\
\hline $\begin{array}{l}\text { 11. Creating } \\
\text { Change }\end{array}$ & 3.97 & 0.61 & & & & & & & & & & & & & & & \\
\hline $\begin{array}{l}\text { 12. Customer } \\
\text { Focus }\end{array}$ & 2.56 & 0.36 & & & & & & & & & & & & & & & \\
\hline $\begin{array}{l}\text { 13. Organizat } \\
\text { ional } \\
\text { Learning }\end{array}$ & 3.73 & 0.55 & & & & & & & & & & & & & & & \\
\hline 14. Mission & 4.13 & 0.68 & & & & & & & & & & & & & & & \\
\hline $\begin{array}{l}\text { 15. Strategic } \\
\text { Direction and } \\
\text { Intent }\end{array}$ & 4.07 & 0.75 & 1 & & & & & & & & & & & & & & \\
\hline $\begin{array}{l}\text { 16. Goals and } \\
\text { Objectives }\end{array}$ & 3.67 & 0.63 & $\begin{array}{l}.91 \\
* * \\
\end{array}$ & 1 & & & & & & & & & & & & & \\
\hline 17. Vision & 3.68 & 0.62 & $\begin{array}{l}.92 \\
* *\end{array}$ & $\begin{array}{l}.97^{*} \\
*\end{array}$ & 1 & & & & & & & & & & & & \\
\hline $\begin{array}{l}\text { 18. Innovatio } \\
\mathrm{n}(\text { sum })\end{array}$ & 1.51 & 4.55 & $\begin{array}{l}.17 \\
* *\end{array}$ & $\begin{array}{c}.16^{*} \\
*\end{array}$ & $\begin{array}{l}.17 \\
* *\end{array}$ & 1 & & & & & & & & & & & \\
\hline $\begin{array}{l}19 . \text { Resource } \\
\text { s made } \\
\text { available } \\
\end{array}$ & 3.70 & 0.67 & $\begin{array}{l}.23 \\
* *\end{array}$ & .178 & $\begin{array}{l}.20 \\
* *\end{array}$ & $\begin{array}{l}.60 \\
* *\end{array}$ & 1 & & & & & & & & & & \\
\hline $\begin{array}{l}\text { 20. Level of } \\
\text { qualifications }\end{array}$ & 3.71 & 1.52 & $\begin{array}{l}.09 \\
*\end{array}$ & $\begin{array}{l}.09 * \\
*\end{array}$ & $\begin{array}{l}.09 \\
*\end{array}$ & $\begin{array}{l}.56 \\
* *\end{array}$ & $\begin{array}{l}.24 \\
* *\end{array}$ & 1 & & & & & & & & & \\
\hline $\begin{array}{l}\text { 21. Lifelong } \\
\text { learning/Trai } \\
\text { ning activities }\end{array}$ & 3.22 & 1.30 & .07 & $.09 *$ & $\begin{array}{l}.09 \\
*\end{array}$ & $\begin{array}{l}.60 \\
* *\end{array}$ & $\begin{array}{l}.30 \\
* *\end{array}$ & .04 & 1 & & & & & & & & \\
\hline
\end{tabular}




\begin{tabular}{|c|c|c|c|c|c|c|c|c|c|c|c|c|c|c|c|c|c|}
\hline $\begin{array}{l}\text { 22. Partnersh } \\
\text { ips and } \\
\text { Cooperation }\end{array}$ & 3.88 & 1.99 & .11 & .04 & .06 & .51 & $\begin{array}{l}.32 \\
* *\end{array}$ & $\begin{array}{l}.30 \\
* *\end{array}$ & $\begin{array}{l}.15 \\
* *\end{array}$ & 1 & & & & & & & \\
\hline $\begin{array}{l}\text { 23. Networki } \\
\text { ng }\end{array}$ & 0.96 & 1.09 & .04 & .03 & .01 & $\begin{array}{l}.42 \\
* * \\
\end{array}$ & $\begin{array}{l}.25 \\
* * \\
\end{array}$ & $\begin{array}{l}.34 \\
* *\end{array}$ & $\begin{array}{l}.10 \\
* * \\
\end{array}$ & $\begin{array}{l}.43 \\
* *\end{array}$ & 1 & & & & & & \\
\hline $\begin{array}{l}\text { 24. Knowled } \\
\text { ge Protection }\end{array}$ & 2.01 & 1.63 & $\begin{array}{c}- \\
.01\end{array}$ & -.05 & $\begin{array}{c}- \\
.04\end{array}$ & $\begin{array}{l}.20 \\
* *\end{array}$ & $\begin{array}{l}.20 \\
* *\end{array}$ & $\begin{array}{l}.17 \\
* *\end{array}$ & $\begin{array}{l}- \\
.16 \\
* *\end{array}$ & .25 & $\begin{array}{l}.21 \\
* *\end{array}$ & 1 & & & & & \\
\hline $\begin{array}{l}\text { 25. Ideas } \\
\text { transformed } \\
\text { into } \\
\text { innovative } \\
\text { projects }\end{array}$ & 2.68 & 0.86 & $\begin{array}{l}.24 \\
* *\end{array}$ & $\begin{array}{c}.20^{*} \\
*\end{array}$ & $\begin{array}{l}.23 \\
* *\end{array}$ & $\begin{array}{l}.34 \\
* *\end{array}$ & $\begin{array}{l}.34 \\
* *\end{array}$ & $\begin{array}{c}- \\
.00\end{array}$ & $\begin{array}{l}.36 \\
* *\end{array}$ & $\begin{array}{l}.09 \\
*\end{array}$ & $\overline{-}$ & $\begin{array}{l}.26 \\
* *\end{array}$ & 1 & & & & \\
\hline $\begin{array}{l}\text { 26. Volume } \\
\text { of new or } \\
\text { improved } \\
\text { products/ser } \\
\text { vices } \\
\end{array}$ & 2.70 & 0.90 & $\begin{array}{l}.24 \\
* *\end{array}$ & $\begin{array}{c}.21 * \\
*\end{array}$ & $\begin{array}{l}.22 \\
* *\end{array}$ & $\begin{array}{l}.45 \\
* *\end{array}$ & $\begin{array}{l}.08 \\
*\end{array}$ & $\begin{array}{l}.07 \\
*\end{array}$ & .11 & .10 & $\begin{array}{c}- \\
.06\end{array}$ & $\begin{array}{c}- \\
.03\end{array}$ & $\begin{array}{l}.26 \\
* *\end{array}$ & 1 & & & \\
\hline $\begin{array}{l}\text { 27. Company } \\
\text { image }\end{array}$ & 4.11 & 0.63 & $\begin{array}{l}.22 \\
* *\end{array}$ & $\begin{array}{c}.15^{*} \\
*\end{array}$ & $\begin{array}{l}.16 \\
* *\end{array}$ & $\begin{array}{l}.47 \\
* *\end{array}$ & $\begin{array}{l}.37 \\
* *\end{array}$ & $\begin{array}{l}.14 \\
* *\end{array}$ & $\begin{array}{c}.07 \\
*\end{array}$ & $\begin{array}{l}.24 \\
* *\end{array}$ & $\begin{array}{l}.19 \\
* *\end{array}$ & $\begin{array}{l}.19 \\
* *\end{array}$ & $\begin{array}{l}.26 \\
* *\end{array}$ & $\begin{array}{l}.39 \\
* *\end{array}$ & 1 & & \\
\hline $\begin{array}{l}\text { 28. Investme } \\
\text { nt in } \\
\text { Research and } \\
\text { Development }\end{array}$ & $\begin{array}{c}59898.2 \\
6\end{array}$ & $\begin{array}{c}588458 . \\
47\end{array}$ & $\begin{array}{l}.19 \\
* *\end{array}$ & $\begin{array}{c}.17^{*} \\
*\end{array}$ & $\begin{array}{l}.19 \\
* *\end{array}$ & $\begin{array}{c}- \\
.03\end{array}$ & $\begin{array}{c}- \\
.00\end{array}$ & $\begin{array}{l}- \\
.14 \\
* *\end{array}$ & $\begin{array}{c}- \\
.04\end{array}$ & $\begin{array}{c}.09 \\
*\end{array}$ & $\begin{array}{c}- \\
.06\end{array}$ & $\begin{array}{l}- \\
0.1\end{array}$ & $\begin{array}{c}- \\
.06\end{array}$ & .00 & $\begin{array}{l}.1 \\
0^{*}\end{array}$ & 1 & \\
\hline $\begin{array}{l}\text { 29. Turnover } \\
\text { Growth }\end{array}$ & $\begin{array}{c}- \\
163628 \\
3.84\end{array}$ & $\begin{array}{l}830458 \\
6.42\end{array}$ & $\begin{array}{c}- \\
.06\end{array}$ & -.03 & $\begin{array}{c}- \\
.05\end{array}$ & $\begin{array}{l}.22 \\
* *\end{array}$ & $\begin{array}{l}- \\
.18 \\
* *\end{array}$ & $\begin{array}{c}.09 \\
*\end{array}$ & $\begin{array}{l}.10 \\
* *\end{array}$ & $\begin{array}{c}- \\
.25 \\
* *\end{array}$ & $\begin{array}{c}- \\
.01\end{array}$ & $\begin{array}{l}- \\
.11 \\
* *\end{array}$ &. & $\begin{array}{c}.09 \\
*\end{array}$ & $\begin{array}{l}- \\
.0 \\
0 \\
\end{array}$ & $\begin{array}{l}'- \\
.5 \\
5 *\end{array}$ & 1 \\
\hline
\end{tabular}

Note:*. Correlation is significant at the 0.05 level (2-tailed).; **. Correlation is significant at the 0.01 level (2tailed)

The structural model was established and the measurement model was specified considering the sector of activity, technology-based companies in one model ( $n=464$ see Figure 1, unconstrained model) and non-technology based companies in another model $(\mathrm{n}=385$; see Figure 2, unconstrained model) tested by multigroup analysis. The results showed a good fit considering NFI=.870 and an acceptable fit for $\mathrm{CFI}=.887$. The score obtained for CMIN/DF (448) was 6.77, p<.001. Considering the RMSEA, the value was .08, which is within the limit of the stipulated requirement for the RMSEA (Schumacker and Lomax, 1996). Considering the criteria mentioned for the quality of the adjustment, it was concluded that the model can be considered adjusted. 


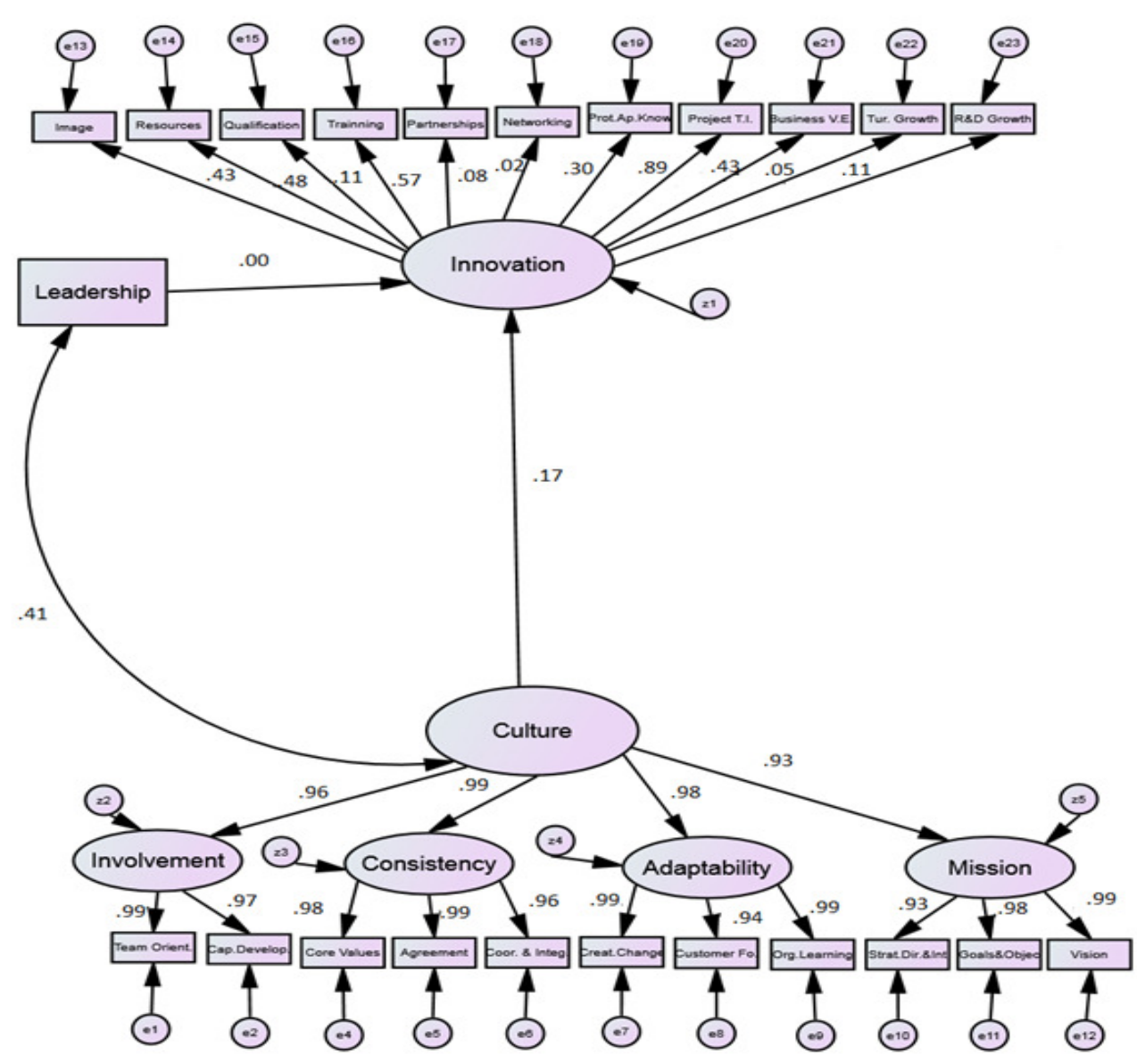

Fig. 1: Innovation predicted by organizational culture and leadership in technologybased companies: Standardized regression coefficients

Legend: Company image (image); resources made available (resources); level of qualification (qualification); training activities (Training); partnerships (partnerships); networks of cooperation (networking); protection and appreciation of knowledge (Prot. Ap. Know); ideas transformed into innovative projects (I.T. Project); sales volume of new or improved products/services (Business V.E); turnover growth (Turn. Growth); Research \& Development growth (R\&D growth); Team Orientation (Team Orient.); Capability Development (Cap. Develop.); Coordination \& Integration (Coor. \& Integ); Creating Change (Creat. Change); Customer Focus (Customer Fo.); Organizational Learning (Org. Learning); Strategic Direction \& Intent (Strat. Dir. \& Int); Goals \& Objectives (Goals \& Objec). 


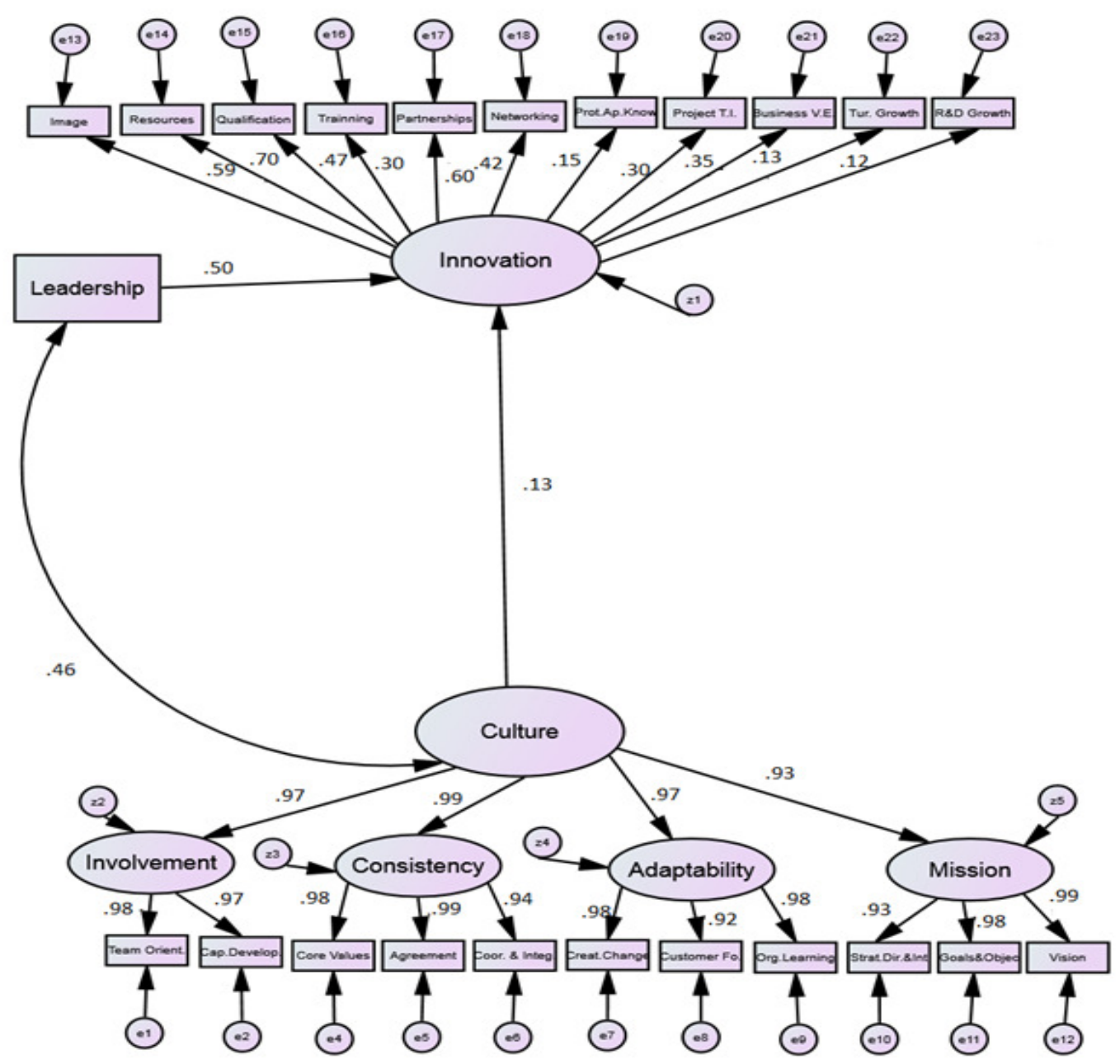

Fig. 2: Innovation predicted by organizational culture and leadership in non-technologybased companies: Standardized regression coefficients

Legend: Company image (image); resources made available (resources); level of qualification (qualification); training activities (Training); partnerships (partnerships); networks of cooperation (networking); protection and appreciation of knowledge (Prot. Ap. Know); ideas transformed into innovative projects (I.T. Project); sales volume of new or improved products/services (Business V.E); turnover growth (Turn. Growth); Research \& Development growth (R\&D growth); Team Orientation (Team Orient.); Capability Development (Cap. Develop.); Coordination \& Integration (Coor. \& Integ); Creating Change (Creat. Change); Customer Focus (Customer Fo.); Organizational Learning (Org. Learning); Strategic Direction \& Intent (Strat. Dir.\& Int); Goals \& Objectives (Goals \& Objec).

The multigroup structural analysis, according to the sector of activity, showed very different regression coefficients for each of the two delimited groups - namely, technology-based companies and nontechnology based companies - which indicate that the sector of activity influences the relationship structure between culture, leadership and innovation (Fig. 1 and Fig. 2).

Analyzing the two models (Fig. 1 and Fig. 2), some of the regression coefficients change the magnitude of the association between the culture, innovation and leadership constructs from one group to 
another: to the same extent, $\lambda \mathrm{ij}$ saturations differ between groups, sometimes oscillating between insignificant and high magnitudes. More specifically, in technology-based companies, the level of qualification was less significant in determining innovation (cf. value of $\lambda=.107)$, while in non-technology-based companies the effect was considerably greater $(\lambda=.474)$. A similar effect occurs with turnover growth $(\lambda=-.05$ for technology-based companies vs. $\lambda=-.13$ for non-technology-based companies), networks $(\lambda=.016$ vs. $\lambda=.423)$ and partnerships $(\lambda=.078 \quad$ vs. $\lambda=.596)$. Comparing between the two models, the simple regression coefficients, it was found that in technology-based companies, the indicators of lifelong learning activities innovation oriented, protection and valorization of knowledge and the sales volume of new or improved products/services were more significant in the promotion of innovation. However, in the non-technology-based companies, the variables resources made available, level of qualification, the partnerships and the networks of cooperation were more significant in the promotion of innovation.

Given the regression coefficients $\beta$, it was found that the effect of leadership on innovation was null for technology-based companies, although it had a magnitude of $\beta=.504$ for non-technology-based companies. This difference highlights the influence of the activity sector on the adopted leadership style, suggesting the activity sector's status as moderating the leadership role in business innovation. In these companies, organizational culture directly determines innovation in a slightly more significant way compared to nontechnology or traditionally based companies. In the case of non-technological companies, the perception of the transformational leadership style was essential in the promotion of innovation, and leadership and organizational culture act together in the promotion of it. In both types of companies' sectors, the association between organizational culture and leadership style was relevant.

\section{Discussion/Conclusion}

This study examines the interrelation between leadership and organizational culture, and their impact on innovation, in technological and non-technological companies. Differences were found between the two theoretical models, showing that the sector of the company acted as a moderator in that relation.

The study showed that the leadership did not have direct effects on innovation in technology based companies but acted indirectly through its organizational culture in innovation. In comparative terms, in a non-technology based company, it was found that the leader acted together with the culture in the promotion of innovation. It can be assumed that the behaviors, attitudes and expectations that explicitly or implicitly arise from the style that characterizes the transformational leader may not match those that are desirable or accepted for the members of technology-based organizations involved in this study. In this case, it may be more effective for the leader to act across the organization's culture to achieve innovation. It can be speculated that other styles of leadership, which are closer to "distributed" or "shared leadership" (Pearce and Sims, 2002) may be more effective in this context than considering only a top-down, hierarchical process. In non-technological companies, the transformational leadership style may prove to be more effective and likely to facilitate change by refocusing the organization's culture towards innovation.

The comparative analysis of innovation indicators allows highlighting some differences between companies in both sectors of activity. The contribution of the qualification level was stronger, in the case of a non-technology based company, which may reflect the effort that these types of companies have made to increase the qualification level of their Human Resources, while the technology-based companies, having more qualified Human Resources (Lousã and Gomes, 2017), has a stronger contribution on employees' participation in lifelong learning activities. 
Indeed, consistent with the literature, the tasks in this latter type of companies are essentially intellectual, analytical, and require training, qualification, experience and creativity to be carried out successfully (Alvesson, 2004; Martins and Terblanche, 2003; Mumford et al., 2002; Burke et al, 2006). Accordingly, employees' competencies are considered as a competitive advantage and raising their competencies enable them to feel that they can act independently. In turn, partnerships and cooperation networks are important in the case of technology-based companies, and not being so relevant in the case of companies in the most technological sector. In the latter, the protection and enhancement of knowledge contributes most to the company's innovation activity. Indeed, while nontechnological companies are more dependent on external relations with other companies to increase their innovation activity, technology-based companies are more dependent on their internal competencies, and as suggested by Trantopoulos, Krogh, Wallin and Woerte (2017), those competencies could have a greater contribution on the companies' innovation process. In terms of results, there is a stronger contribution on the ideas that are transformed into innovative projects and the evolution of sales volume of new or significantly improved products and/or services in the case of technologybased companies, while in other companies, the innovation activity is more related to its image and reputation. One difference that deserves to be highlighted concerns a greater contribution to innovation activities at the company's resource level or inputs in the nontechnological sector, while in the technology-based sector, innovation is translated into better company results.

A contribution of the present study is that transformational leaders could act directly or indirectly, creating or developing the culture of their organization to promote innovation. Also, it may provide contributions for managers to evaluate or explore their organization innovation activity and to identify areas of improvement within and across different industries or companies.

\section{Limitations and future directions}

Despite contributions, some limitations to the present study are noteworthy and may be useful for future research directions. The use of a non-probability design sampling limits the generalizability of the findings of the study for all the sectors of activity. Also, the moderation effects of the activity sector need to be assessed from different industry groupings that will reflect more diverse technological contexts. Future research should investigate other populations with a probabilistic sample, thus generating more plausible generalization results. The cross-sectional character of the research method also represents another limitation to the present study, since it is based on the analysis of a single moment. Future longitudinal studies could advance new discoveries in the field. The companies of the sample operate within Portugal; therefore, some caution should be taken in extrapolating the results to other countries. This could be a future avenue for research.

\section{References}

- Amabile, T. M., Conti, R., Coon, H., Lazenby, J., and Herron, M. (1996), 'Assessing the work environment for creativity', Academy of Management Journal, 39 (5), 1154-1184.

- Bass, B. M. (1999), 'Two decades of research and development in transformational leadership', European Journal of Work and Organizational Psychology, 8 (1), 9-32.

- Bass, B., M. and Avolio J. B. (1993), 'Transformational leadership and organizational culture', Public Administration Quarterly, spring, 112121.

- Bass, B. M., and Riggio, R. E. (2006), Transformational leadership (2 ed.), Mahwah, NJ: Lawrence Erlbaum.

- Bollen, K.A. (1989), Structural equations with latent variables, New York: Wiley. 
- Byrne, B. M. (2010), Structural equation modeling with AMOS: Basic concepts, applications and programming (2nd ed.). London: Lawrence Erlbaum.

- Burke, C. S., Stagl, K. C., Klein, C., Goodwin, G. F., Salas, E., and Halpin, S. M. (2006), 'What type of leadership behaviors are functional in teams? A meta-analysis', The leadership quarterly, 17(3), 288-307.

- $\quad$ Chandler, G. N., Keller, C., and Lyon, D. W. (2000), 'Unravelling the determinants and consequences of an innovation-supportive organizational culture', Entrepreneurship Theory and Practice, 25(1), 59-76.

- Cohen, J. (1988), Statistical power analysis for the behavioural sciences (2nd ed.), New York: Academic Press.

- Damanpour, F., and Wischnevsky, D. (2006), 'Research on innovation in organizations: Distinguishing innovation-generating from innovation-adopting organizations', Journal of Engineering and Technology Management, 23(4), 269-291.

- Denison, D., Nieminen, L. and Kotrba, L. (2014), 'Diagnosing organizational cultures: A conceptual and empirical review of culture effectiveness surveys', European Journal of Work and Organizational Psychology, 23 (1), 145161.

- Gumusluoglu, L., and Ilsev, A. (2009), 'Transformational leadership, creativity, and organizational innovation', Journal of Business Research, 62, 461-473.

- Hoyle, R. (1995), Structural equation modeling: Concepts, issues, and applications, Thousand Oaks, CA: Sage Publications.

- Jung, D. I., Chow, C., and Wu, A. (2003), 'The Role of Transformational Leadership in Enhancing Organizational Innovation: Hypotheses and Some Preliminary Findings', Leadership Quarterly, 14, 525-544.

- Jung, D. I., Wu, A., and Chow, C. (2008), 'Towards understanding the direct and indirect effects of CEOs' transformational leadership on firm
Innovation', Leadership Quarterly, 19, 582-594.

- Loehlin, J. C. (1997), Latent variable models, Mahwah, NJ: Lawrence Erlbaum Associates.

- Lousã, E. P. (2013), Liderança empreendedora e cultura de inovação em organizações de base tecnológica e análise comparativa entre setores de atividade, A Dissertation submitted in fulfillment of the requirements of the doctor of philosophy, University of Coimbra, Faculty of Psychology and Education Sciences, Portugal.

- Lousã, E.P. and Gomes, A. D. (2017), 'The influence of technology, organisational size and age on Innovation', Revista Psicologia: Organizações e Trabalho, 17 (4), 252259.

- Lousã, E.P. and Mónico, L. S. M. (2018)," How can leadership and organizational culture predict innovation in small, medium and large enterprises?", The Journal of Organizational Management Studies, Vol. 2018 (2018), 1-15 Article ID 703891, DOI: $10.5171 / 2018.703891$

- Martins, E. C. and Terblanche, F. (2003), 'Building Organizational Culture That Stimulates Creativity and Innovation', European Journal of Innovation Management, 6(1), 64-74

- Mumford, M. D., Scott, G. M., Gladdis, B., and Strange, J. M. (2002), 'Leading creative people: orchestrating expertise and relationships', Leadership Quarterly, 13, 705-750.

- OECD (2005), Oslo Manual: Guidelines for collecting and Interpreting Innovation Data, Paris: Organisation for Economic Co-operation and Development.

- OECD (2015), OECD Innovation Strategy 2015: An Agenda for Policy Action, Paris: Organisation for Economic Co-operation and Development.

- Ramos, J., Anderson, N., Peiró, J. M., and Zijlstra, F. (2016), 'Studying innovation in organizations: a dialectic perspective - introduction to the special issue', European Journal of 
Work and Organizational Psychology, 25(4), 477-480.

- Schumacker, R. E., and Lomax, R. G. (2010), A beginner's guide to structural equation modeling (3rd ed.), Mahwah, N.J.: Lawrence Erlbaum Associates.

- Scott, S. G., and Bruce, R. A. (1994), 'Determinants of innovative behavior: a path model of individual innovation in the workplace', Academy of Management Journal, 37, 580-607.

- Trantopoulos, K., Krogh, G. V., Wallin, M., and Woerter, M. (2017), 'External knowledge and information technology: Implications for process innovation performance'. Management Information Systems Quarterly, 41(1), 287-300. 